\title{
Is there a role for fractional flow reserve in coronary artery bypass graft (CABG) planning?
}

\author{
Amir Ahmadi ${ }^{1,2}$, Dylan Stanger ${ }^{2}$, John Puskas $^{1}$, David Taggart ${ }^{3}$, Y. Chandrashekhar ${ }^{4}$ Jagat Narula $^{1}$ \\ ${ }^{1}$ Mount Sinai Heart, Icahn School of Medicine at Mount Sinai Hospital, New York, New York, USA; ${ }^{2}$ Division of Cardiology, Department of \\ Medicine, University of British Columbia, Vancouver, British Columbia, Canada; ${ }^{3}$ Nuffield Department of Surgical Sciences, University of Oxford, \\ Oxford, UK; ${ }^{4}$ Department of Cardiology, Veterans Administration Hospital and University of Minnesota School of Medicine, Minneapolis, USA \\ Correspondence to: Jagat Narula, MD. Mount Sinai Heart, Icahn School of Medicine at Mount Sinai Hospital, New York, New York, USA. \\ Email: Narula@mountsinai.org.
}

\begin{abstract}
The concept of significant lesions has substantially evolved over the last decade. With growing evidence for use of fractional flow reserve (FFR) as a determinant of lesion-specific ischemia and its superiority to angiography-guided revascularization and medical therapy, the field of percutaneous revascularization has shifted to rely exclusively on FFR instead of luminal stenosis alone in guiding revascularization. This transition to physiological assessment has not yet made it to the realm of surgical revascularization. FFRguided therapy has been shown to be superior to angiography-guided therapy mainly by safe deferral of about $1 / 3$ rd of lesions, leading to less periprocedural events and better outcomes. Is it possible that utilization of FFR-guided CABG would lead to less complicated procedures, shorter operating times, more frequent off pump CABG procedures and more hybrid procedures? Can FFR-guided CABG improve the cardiovascular outcomes as compared to current standard of practice? In the following paragraphs we review the concept of FFR, the evidence behind FFR-guided therapy, the emerging data regarding use FFR-guided CABG and discuss where the revascularization field is headed.
\end{abstract}

Keywords: Fractional flow reserve; coronary artery bypass grafting (CABG); coronary revascularisation; FFRguided $\mathrm{CABG}$

Submitted Jul 11, 2018. Accepted for publication Jul 12, 2018.

doi: 10.21037/acs.2018.07.01

View this article at: http://dx.doi.org/10.21037/acs.2018.07.01

\section{Introduction}

Coronary artery bypass grafting (CABG) has been performed in a uniform way for over 50 years, with preoperative invasive coronary angiography visually assessed by cardiologists and utilized by surgeons to determine the target epicardial vessels to be bypassed (1). This decision was made based on anatomic stenoses of $50 \%$ or greater of the left main coronary artery, and usually $70 \%$ or greater anatomic stenosis for the other main epicardial vessels (1).

Conventional coronary angiography identifies luminally obstructive coronary artery disease which is often equated with hemodynamically significant stenoses (2) without robust scrutiny. A number of studies have shown this faith in angiography is unfounded, both when compared with quantitative coronary angiography (QCA) (3) or more recently, with more robust methods of determining the physiologic significance of any given angiographic stenosis, like fractional flow reserve (FFR) (4). FFR measurement has been well validated as a better invasive means of assessing the physiologic significance of an epicardial coronary artery stenosis. With time, FFR has substantially changed our understanding of the complex interplay between anatomical stenoses and functional flow limitations (5). The Fractional Flow Reserve Versus Angiography for Multivessel Evaluation (FAME) study demonstrated the superiority of FFR-guided therapy to conventional angiography in guiding percutaneous coronary revascularization, primarily by safe deferral of lesions that were "stenotic" but did not result in lesion specific ischemia by FFR. Contemporary societal 
guidelines support the use of FFR to complement coronary angiography, with the highest degree of recommendation for the assessment of coronary stenosis before implementing coronary revascularization (when previous non-invasive functional evaluation is not available or is inconclusive) (6). Many argue that FFR should also play an important role in risk stratification and determination of management strategy of patients either before or after CABG as well. However, the utility of FFR in CABG remains poorly studied (7) and the consequences of closure of a bypass graft versus closure of a coronary stent in the setting of borderline native coronary stenosis may be very different.

\section{Modern understanding of FFR physiology}

Understanding the physiology of epicardial artery blood flow is critical to understanding the use of FFR. While luminal stenosis is a known predictor of ischemia, the stenosis-ischemia relationship is far from perfect (8). There are a considerable number of lesions that are stenotic without (causing) ischemia (SWOI) and ones that are ischemic without significant stenosis (IWOS). The imperfect relationship between stenosis and ischemia has been traditionally explained by limitations of $2 \mathrm{D}$ interpretation of invasive coronary angiography (ICA). However, more accurate measures of luminal narrowing such as minimal luminal diameter (MLD) and minimal luminal area (MLA) with intravascular ultrasound (IVUS) did not succeed in strengthening the ischemia-stenosis agreement (9). Subsequently, other anatomic characteristics such as the lesion length, entrance and exit angles, and the size of reference vessel were invoked to explain the discrepancy (10). Recently, it has been demonstrated that low attenuation plaque (LAP) volume on CTA (surrogate for necrotic core) is a determinant of lesion specific ischemia by FFR, independent of luminal stenosis. In fact, amongst all lesion characteristics, LAP volume along with the degree of luminal stenosis were the only two lesion factors that were independent predictors of FFR in a multivariate model (11-13).

In order to understand the importance of necrotic core volume in determination of FFR, it is crucial to recognize that FFR is not a static measure and coronary arteries are not rigid pipes. In addition to the fixed degree of stenosis, a vessel's behavior at the time of achieving maximal hyperemia has a major role in determining post stenotic pressure measurement. The infusion of adenosine during the FFR measurement dilates the distal arteriolar bed which in turn drops the pressure in that region. This drop in pressure causes a larger gradient to be developed between the aorta and distal coronary bed, increasing the coronary blood flow (state of maximal hyperemia). Epicardial coronary artery auto-regulatory mechanisms respond to the state of maximal hyperemia by further dilatation which is exacerbated by the administration of nitroglycerine as a routine part of the protocol for obtaining the FFR. In the classic case of severe luminal stenosis, since the vessel at the level of stenosis is at the maximally dilated state at rest, it cannot dilate any further. Therefore, with maximal hyperemia, this inability to accommodate for the extra blood flow will result in a post stenotic pressure drop at the time of hyperemia (13).

In the case of mild to moderate luminal stenosis, a large necrotic core and the respective vessel maximally stretched outwards (positive remodeling), it is hypothesized that the vessel likely develops a degree of impairment in its ability to dilate locally at the lesion site. With the rest of the vessel dilated during maximal hyperemia, the mild to moderate stenotic region becomes a functionally significant stenosis (a relative significant stenosis). Given trans-stenotic pressure drop is inversely proportional to the fourth power of the lumen's radius, the physiologic significance of any fixed or dynamic narrowing is defined by a curvilinear pressure-flow relationship (14). As a consequence, a relative significant stenosis produces a marked hemodynamic effect and an abnormal FFR measurement.

FFR could be thought of as a sensitive but not specific detector of high risk plaque (with a large necrotic core). Not all FFR+ lesions are high risk lesions, but most FFR lesions are devoid of a large necrotic core. Due to these facts, FFR negative lesions have favorable prognosis- independent of luminal stenosis. Given that it has been shown repeatedly that (I) percutaneous revascularization based on anatomical stenosis is not superior to optimal medical therapy, (II) ischemia is an important predictor of future outcomes and should guide revascularization and (III) most myocardial infarctions happen as a result of a plaque with high risk features; the utility of FFR in addition (or in lieu) of the degree of luminal stenosis for guiding revascularization seems to be very logical, as it gives information on the three key features of a lesion, namely luminal stenosis, ischemia, and plaque morphology.

\section{FFR-guided revascularization and outcomes}

Prior to FFR being commonly utilized in clinical practice, 
patients undergoing invasive coronary angiography without prior documented ischemia on non-invasive testing could potentially undergo revascularization of an anatomically stenosed lesion without being certain of a future clinical benefit. Over past years, considerable evidence has accrued supporting functional revascularization in stable ischemic heart disease (SIHD) (7). The FAME trial (5) demonstrated that in patients with SIHD, an FFR-guided strategy to identify hemodynamically significant lesions requiring percutaneous coronary intervention (PCI) can safely defer percutaneous revascularization in lower-risk lesions. It also showed that this strategy reduced the number of procedures and rates of future urgent revascularization due to unstable angina or myocardial infarction (MI) compared with lesion selection by angiography alone. In the FAME study, more than one-third of lesions with an angiographic $50 \%$ to $70 \%$ angiographic diameter stenosis demonstrated an FFR of 0.80 or less whereas one-fifth of lesions with a $71 \%$ to $90 \%$ angiographic diameter stenosis demonstrated an FFR greater than 0.80 . These findings underscore the importance of identifying factors beyond luminal stenosis that might contribute to inducible ischemia $(11,13)$. The FAME2 trial extended the aforementioned findings and demonstrated that deferring PCI in lesions with an abnormal FFR results in high rates of progressive ischemic symptoms, and unstable angina leading to urgent revascularization within 1, 2, 3 and 5 years of follow-up (15).

While there is a shift in decision-making strategy for revascularization by PCI to involve functional testing and lesion specific ischemia by FFR, revascularization by CABG has continued to be based on anatomic assessment alone. As our understanding of the concept of the "at risk lesion" is evolving beyond presence of luminal stenosis alone and involves concepts such as ischemia, plaque morphology and vessel's vasodilatory ability, it might be the time to reevaluate how we define "significant coronary artery disease/stenosis" requiring revascularization by CABG.

\section{FFR utilization and CABG}

Because visual assessment of a stenosis has been found to have $\pm 20 \%$ variance, occasionally, milder lesions are bypassed with the rationale that if coronary disease progressed, the bypass graft would end up serving a protective role (16). This concept has been fraught with significant controversy and refuted in several studies that have demonstrated if functionally insignificant lesions are bypassed, early graft failure is occasionally seen and acceleration of native artery disease potentially exacerbates the consequences of late graft failure (16). Previous work has suggested that that up to $25 \%$ of bypass grafts to vessels that are not physiologically stenosed actually provide no measurable perfusion to the territory (17).

There are data that suggest $<50 \%$ of patients who were thought to have multi-vessel disease causing ischemia actually have functionally significant multivessel disease (MVD) (18). In contemporary practice, patients found to have LM or MVD on invasive coronary angiography are referred for CABG surgery. Extrapolating from this information, it would be expected that occasionally, some bypasses will be grafted to native vessels which do not have functionally significant lesions (18). When a moderately diseased native vessel is bypassed, the perfusion result may not be effective. In fact, the final result may even be harmful. There is an evolving body of evidence that suggests an accelerated atherosclerotic process may be propagated in native vessels, especially those which are bypassed with venous grafts. However, there is also data that suggests that there is no change in mortality (17).

The interesting question of whether randomized FFRguided PCI data extends to CABG populations is of utmost relevance. In a 2013 observational registry study by Toth et al., FFR-guided coronary artery bypass graft surgery was found to be associated with a lower number of graft anastomoses and a lower rate of on-pump surgery compared with angiography-guided coronary artery bypass graft surgery. This did not result in a change in event rate during up to 36 months of follow-up and was associated with a lower rate of angina (19).

The same group published long term outcomes and showed that FFR-guided CABG was associated with a significant reduction in the rate of overall death or myocardial infarction at 6-year follow-up as compared with angiography-guided CABG. This study enrolled over 600 patients who were treated with CABG. At least 1 stenosis was grafted according to FFR, whereas the other group had stenoses grafted based on angiography. The two co-primary endpoints were overall death or MI and major adverse cardiovascular events. The rate of overall death or MI was significantly lower in the FFR-guided group compared to the angiography group (7).

The recently published SYNTAX III Revolution trial aimed to evaluate whether a Heart Team decision-making process regarding the choice of revascularization strategy based on non-invasive coronary CT angiography assessment of CAD is equivalent to the standard-of-care invasive 
angiography-based assessment in patients with multivessel CAD. The trial showed that in patients with left main or three-vessel coronary artery disease, a heart team treatment decision-making based on coronary CTA with $\mathrm{FFR}_{\mathrm{CT}}$ showed an almost perfect agreement with the decision derived from conventional coronary angiography. This suggests the potential feasibility for treatment decisionmaking and planning based solely on this non-invasive imaging modality (20).

The reliance on epicardial anatomy has led to the development of numerous assumptions about CABG (1). These assumptions include (but are not limited to) the idea that placement of a technically satisfactory graft beyond a significant lesion will result in increased blood flow and reperfusion to surrounding myocardium. However, the whole concept of what is a significant lesion, which lesions need revascularization and which are safe with medical therapy is evolving. With ease of access to non-invasive ischemia testing, FFR and plaque morphology assessment (both invasively and non-invasively), decision making regarding revascularization is moving to a much more complete and complex assessment of the lesion as a whole rather than being solely stenosis-based (21).

FFR-guided CABG is emerging as an important tool in cardiac surgery, with anatomic, functional and even morphological characteristics of the target vessel and coronary artery stenosis able to optimize graft site selection (13). The surgeon should consider that the physiologic response to bypass grafting may well be determined by the type of lesion (11).

Invasive, functionally-guided surgical revascularization has never been prospectively evaluated. The GRAFFITI trial is a RCT currently evaluating FFR-guided versus angiography-guided CABG. It is expected to be completed soon and may provide randomized data to help guide this decision-making process (22).

The FAME 3 trial is also underway. It is a multicentre, world-wide, prospective randomized controlled trial with a non-inferiority design. It will compare FFR-guided with coronary angiography-guided PCI using $2^{\text {nd }}$ generation resolute drug-eluting stents in patients with multivessel coronary artery disease. The investigator's hypothesis is that FFR-guided PCI with $2^{\text {nd }}$ generation stents will result in similar outcomes to CABG in patients with multivessel disease. The study design currently calls for 2 years of enrollment and up to 5-year follow-up. The estimated study completion date is August 2021.

Given the momentum behind FFR and ongoing and future randomized data looking at utilization of FFR- guided CABG, it is likely that we will be moving away from an anatomical assessment to a functional assessment to inform revascularization decisions for CABG. One important issue to remember, when extrapolating data regarding the use of FFR in PCI to CABG while waiting for formal randomized trials in CABG itself, is that such PCI-FFR studies excluded typical patients who would be coming for $\mathrm{CABG}$ in any given clinical practice: patients with CTOs, complex multi vessel disease not best suited for PCI or those with associated valve disease. Moreover, CTO and extensive collateralized beds negatively influence FFR. In addition, is it important to remember that the large randomized comparisons made between angiographyguided PCI versus angiography-guided CABG (i.e., the FREEDOM, SYNTAX and NOBLE trial) (23-25) found that patients with left main and three vessel diseases had better long-term outcomes with CABG than PCI, especially in the setting of diabetes. This may partly be because of the relatively benign nature of graft failure compared to stent failure in coronary arteries with limited native stenosis. Another possible explanation may be that a bypass graft protects the patient from present coronary lesion(s) and future lesions that may develop proximal to the graft. Finally, arterial grafts, especially internal mammary grafts, are associated with lower rates of graft failure and native coronary occlusion than venous grafts and their increased use should be a common goal of cardiologists and surgeons. A recent patient-level meta-analysis demonstrated that the addition of a radial arterial conduit to a LIMA-plusvein-grafts strategy leads to significantly improved clinical outcomes for CABG patients (22).

\section{Non-invasive assessment of FFR in CABG planning}

CTA is an excellent tool to manage patients with chronic stable angina even in routine clinical practice (26). It carries the advantage of being able to assess the need for invasive angiography and so acts as an efficient gate keeper to minimize non-productive invasive angiography (i.e., angiography that does not result in meaningful intervention after the procedure) (27). It comes with the added advantage of being well-correlated with invasive FFR, noninvasive, easily repeatable and takes data sets from routinely obtained CTA studies (meaning no extra effort is needed to incorporate this into practice). It can now be done on desktop systems (28). This is likely to radically change the paradigm of how we assess coronary stenosis and who we send for percutaneous intervention. How this will affect our 
decision-making process for CABG remains to be seen.

A further post-operative advantage of CTA is accurate detection of post-CABG graft failure (29)—the large size of the venous grafts, and less motion make this technique more accurate in detecting stenosis in grafts than in native vessels distal to the grafts. It also has prognostic predictive value (30). While there is little data about use of CT-FFR in graft disease (31) it is only a matter of time before this will be studied extensively in decision making for re-do surgery.

\section{Future trends}

There is mounting evidence supporting the concept that FFR guidance reduces the number of lesions requiring revascularization. Due to these advances, we anticipate there will be numerous potential benefits for patients with respect to surgical revascularization. Specifically, we anticipate there will shorter surgical wait times as well as more off-pump and robotic CABG interventions. Additionally, we expect that there will be in an increased role for hybrid procedures involving single-vessel CABG with PCI to other lesions. With fewer vessels requiring $\mathrm{CABG}$, it is anticipated there will be a higher overall percentage of arterial grafts utilized. The authors strongly believe that LIMA to LAD grafting is essential to optimal CABG outcomes and the PCI and FFR data are more favourable for the non-LAD territories, especially when compared to venous bypass grafts.

Furthermore, the fundamental question to ask with any lesion being considered for revascularization is whether the goal is to improve anginal symptoms or decrease events. PCSK9 inhibitors have been demonstrated to reduce plaque volume and necrotic core volume and reduce cardiovascular events but also reverse ischemia (32). It is therefore imperative that maximal medical therapy be duly instituted before and after FFR assessment and revascularization. Indeed, many randomized trials have shown lower rates of optimal medical management in the CABG groups than the PCI groups $(23,24,33)$; we emphasize the importance of optimal medical management in all patients with coronary artery disease, especially after invasive revascularization. The power of selective revascularization in conjunction with contemporary medical therapies promises to optimize patient outcomes more than ever before.

\section{Acknowledgements}

None.

\section{Footnote}

Conflicts of Interest: The authors have no conflicts of interest to declare.

\section{References}

1. ElBardissi AW, Aranki SF, Sheng S, et al. Trends in isolated coronary artery bypass grafting: an analysis of the Society of Thoracic Surgeons adult cardiac surgery database. J Thorac Cardiovasc Surg 2012;143:273-81.

2. Bundhun PK, Yanamala CM, Huang F. Comparing the adverse clinical outcomes associated with fraction flow reserve-guided versus angiography-guided percutaneous coronary intervention: a systematic review and metaanalysis of randomized controlled trials. BMC Cardiovasc Disord 2016;16:249.

3. White CW, Wright CB, Doty DB, et al. Does visual interpretation of the coronary arteriogram predict the physiologic importance of a coronary stenosis? N Engl J Med 1984;310:819-24.

4. Tonino PA, Fearon WF, De Bruyne B, et al. Angiographic versus functional severity of coronary artery stenoses in the FAME study fractional flow reserve versus angiography in multivessel evaluation. J Am Coll Cardiol 2010;55:2816-21.

5. Tonino PA, De Bruyne B, Pijls NH, et al. Fractional flow reserve versus angiography for guiding percutaneous coronary intervention. N Engl J Med 2009;360:213-24.

6. Patel MR, Dehmer GJ, Hirshfeld JW, et al. ACCF/ SCAI/STS/AATS/AHA/ASNC 2009 Appropriateness Criteria for Coronary Revascularization: A Report of the American College of Cardiology Foundation Appropriateness Criteria Task Force, Society for Cardiovascular Angiography and Interventions, Society of Thoracic Surgeons, American Association for Thoracic Surgery, American Heart Association, and the American Society of Nuclear Cardiology: Endorsed by the American Society of Echocardiography, the Heart Failure Society of America, and the Society of Cardiovascular Computed Tomography. Circulation 2009;119:1330-52.

7. Fournier S, Toth GG, De Bruyne B, et al. Six-Year Follow-Up of Fractional Flow Reserve-Guided Versus Angiography-Guided Coronary Artery Bypass Graft Surgery. Circ Cardiovasc Interv 2018;11:e006368.

8. Ahmadi A, Kini A, Narula J. Discordance between ischemia and stenosis, or PINSS and NIPSS: are we ready for new vocabulary? JACC Cardiovasc Imaging 2015;8:111-4. 
9. Johnson NP, Kirkeeide RL, Gould KL. Coronary anatomy to predict physiology: fundamental limits. Circ Cardiovasc Imaging 2013;6:817-32.

10. Kern MJ, Samady H. Current concepts of integrated coronary physiology in the catheterization laboratory. J Am Coll Cardiol 2010;55:173-85.

11. Ahmadi A, Stone GW, Leipsic J, et al. Association of coronary stenosis and plaque morphology with fractional flow reserve and outcomes. JAMA Cardiol 2016;1:350-7.

12. Gaur S, Ovrehus KA, Dey D, et al. Coronary plaque quantification and fractional flow reserve by coronary computed tomography angiography identify ischaemiacausing lesions. Eur Heart J 2016;37:1220-7.

13. Ahmadi A, Leipsic J, Ovrehus KA, et al. Lesion-Specific and Vessel-Related Determinants of Fractional Flow Reserve Beyond Coronary Artery Stenosis. JACC Cardiovasc Imaging 2018;11:521-30.

14. Spaan JA, Piek JJ, Hoffman JI, et al. Physiological basis of clinically used coronary hemodynamic indices. Circulation 2006;113:446-55.

15. De Bruyne B, Pijls NH, Kalesan B, et al. Fractional flow reserve-guided PCI versus medical therapy in stable coronary disease. N Engl J Med 2012;367:991-1001.

16. Botman CJ, Schonberger J, Koolen S, et al. Does stenosis severity of native vessels influence bypass graft patency? A prospective fractional flow reserve-guided study. Ann Thorac Surg 2007;83:2093-7.

17. Dauerman HL. Reasonable incomplete revascularization. Circulation 2011;123:2337-40.

18. Ferguson TB Jr, Chen C, Buch AN. Fractional flow reserve-guided coronary bypass surgery: should surgeons use it? Curr Opin Cardiol 2013;28:654-60.

19. Toth G, De Bruyne B, Casselman F, et al. Fractional flow reserve-guided versus angiography-guided coronary artery bypass graft surgery. Circulation 2013;128:1405-11.

20. Coronary Computed Tomography Angiography for Heart Team Decision-Making in Multivessel Coronary Artery Disease: The SYNTAX III REVOLUTION Trial. Available online: https://solaci.org/_files/pcr2018/Syntax3-Revolution-presentacion.pdf

21. Ahmadi A, Stone GW, Leipsic J, et al. Prognostic Determinants of Coronary Atherosclerosis in Stable Ischemic Heart Disease: Anatomy, Physiology, or Morphology? Circ Res 2016;119:317-29.

22. Ahmadi A, Narula J. Primary and Secondary Prevention, or Subclinical and Clinical Atherosclerosis. JACC Cardiovasc Imaging 2017;10:447-50.

23. Farkouh ME, Dangas G, Leon MB, et al. Design of the
Future REvascularization Evaluation in patients with Diabetes mellitus: Optimal management of Multivessel disease (FREEDOM) Trial. Am Heart J 2008;155:215-23.

24. Serruys PW, Onuma Y, Garg S, et al. Assessment of the SYNTAX score in the Syntax study. EuroIntervention 2009;5:50-6.

25. Makikallio T, Holm NR, Lindsay M, et al. Percutaneous coronary angioplasty versus coronary artery bypass grafting in treatment of unprotected left main stenosis (NOBLE): a prospective, randomised, open-label, non-inferiority trial. Lancet 2016;388:2743-52.

26. Norgaard BL, Hjort J, Gaur S, et al. Clinical Use of Coronary CTA-Derived FFR for Decision-Making in Stable CAD. JACC Cardiovasc Imaging 2017;10:541-50.

27. Curzen NP, Nolan J, Zaman AG, et al. Does the Routine Availability of CT-Derived FFR Influence Management of Patients With Stable Chest Pain Compared to CT Angiography Alone?: The FFRCT RIPCORD Study. JACC Cardiovasc Imaging 2016;9:1188-94.

28. Kruk M, Wardziak L, Demkow M, et al. WorkstationBased Calculation of CTA-Based FFR for Intermediate Stenosis. JACC Cardiovasc Imaging 2016;9:690-9.

29. Barbero U, Iannaccone M, d'Ascenzo F, et al. 64 slicecoronary computed tomography sensitivity and specificity in the evaluation of coronary artery bypass graft stenosis: A meta-analysis. Int J Cardiol 2016;216:52-7.

30. Mushtaq S, Andreini D, Pontone G, et al. Prognostic value of coronary CTA in coronary bypass patients: a long-term follow-up study. JACC Cardiovasc Imaging 2014;7:580-9.

31. Di Serafino L, De Bruyne B, Mangiacapra F, et al. Longterm clinical outcome after fractional flow reserve- versus angio-guided percutaneous coronary intervention in patients with intermediate stenosis of coronary artery bypass grafts. Am Heart J 2013;166:110-8.

32. Sabatine MS, Giugliano RP, Keech AC, et al. Evolocumab and Clinical Outcomes in Patients with Cardiovascular Disease. N Engl J Med 2017;376:1713-22.

33. Stone GW, Sabik JF, Serruys PW, et al. EverolimusEluting Stents or Bypass Surgery for Left Main Coronary Artery Disease. N Engl J Med 2016;375:2223-35.

Cite this article as: Ahmadi A, Stanger D, Puskas J, Taggart D, Chandrashekhar Y, Narula J. Is there a role for fractional flow reserve in coronary artery bypass graft (CABG) planning? Ann Cardiothorac Surg 2018;7(4):546-551. doi: 10.21037/ acs.2018.07.01 